\title{
Novel strategies in vaccine design: can nanocapsules help prevent and treat hepatitis $B$ ?
}

\author{
"Virus-derived nanoparticles are mainly attractive for \\ innovative vaccine formulations, enabling the development \\ of robust and biodegradable protein nanocapsules made \\ of the pathogen-specific antigen.'
}

First draft submitted: 20 February 2017; Accepted for publication: 15 March 2017; Published online: 18 May 2017

Keywords: co-administration of vaccine-antigen and -adjuvant $\bullet$ hepatitis B $\bullet$ nanocapsules - nanoparticulate $\bullet$ vaccination $\bullet$ vaccine

\section{Current status regarding hepatitis B vaccine}

In general, current immunization strategies against hepatitis $\mathrm{B}$ virus $(\mathrm{HBV})$ are safe and efficient ( $>95 \%$ protection) [1]. The available US FDA approved HBV vaccines require three- or four-dose series over a 6-to 12 -month period to obtain the most effective protection [2]. These formulations are based on recombinant hepatitis $B$ surface antigen ( $H B s A g)$, which self-assembles into immunogenic virus-like particles (VLPs) [3]. VLPs consist of the virus capsid or envelope proteins that mimic the organization of the native viruses but lack the viral genome [4]. In contrast to live-attenuated virus vaccines, VLPs are unable to replicate which attributes to their high safety profile. So far, there are only few VLP-based vaccines on the market. These include the above-mentioned prophylactic vaccines against $\mathrm{HBV}$ (e.g., Engerix ${ }^{\circledR}$ ) along with Cervarix ${ }^{\circledR}$ and Gardasil ${ }^{\circledR}$ against the human papillomavirus (HPV) [5]. Unfortunately, recombinant proteins display a limited immunogenicity and require additional adjuvants to increase the level of protection [6]. Likely the oldest and most widely used vaccine adjuvant is aluminium hydroxide or -phosphate, referred to as alum [7]. The mechanism of action behind the use of alum as an adjuvant has been poorly understood until a few years ago $[8,9]$. It has been shown that alum activates the NLR family, pyrin domain containing 3 (NLRP3) inflammasone subsequently leading to the release of immune mediators and the activation of antigen-presenting cells (APCs) [9]. Immunization with alum-based hepatitis $B$ vaccines induce a humoral $\mathrm{T}_{\mathrm{H}}$ 2-directed immune response, which results in the generation of neutralizing antibodies against $H B s A g$ and viral clearance during the acute phase of infection [10]. In contrast, the eradication of chronic HBV infections relies on the induction of robust $\mathrm{CD}^{+}$and $\mathrm{CD} 8^{+} \mathrm{T}$-cell responses [11,12]. Thus, alum-based vaccines are mainly suitable for prophylactic vaccination in industrialized countries, which have the access to a regulated healthcare with a universal immunization program. Individuals in endemic countries often do not have access to three consecutive immunizations [13]. Consequently, single-dose HBV vaccines that induce $T_{H}$ 1-type responses inherit the potential to significantly promote adherence to immunization and protection against HBV infection [14].

\section{Current limitations of HBV vaccines}

The rather complex immunization schedule with at least two booster immunizations limits the applicability of HBV vaccines, particularly in countries with limited healthcare resources. The implementation of hepatitis B
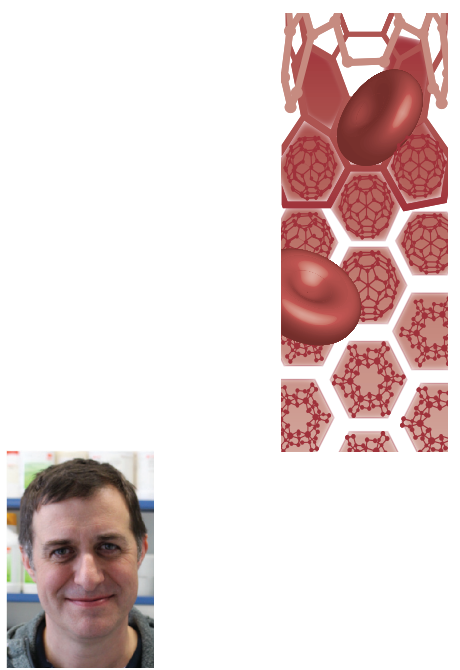

Stephan Gehring Author for correspondence: Children's Hospital, University Medical Center, Johannes Gutenberg University, 55128 Mainz, Germany stephan.gehring@uni-mainz.de

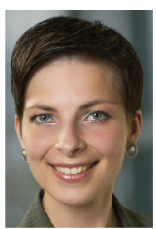

Anette Pietrzak-Nguyen Children's Hospital, University Medical Center, Johannes Gutenberg University, 55128 Mainz, Germany

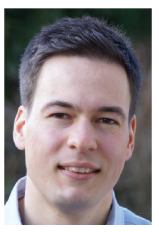

Michael Fichter

Children's Hospital, University Medical Center, Johannes Gutenberg University, 55128 Mainz, Germany

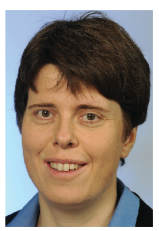

Katharina Landfester Author for correspondence: Max Planck Institute for Polymer Research, Ackermanweg 10, 55122 Mainz, Germany landfester@mpip-mainz.mpg.de

Future $\because$ Medicine part of 
vaccines requires more effort to overcome the social and economic challenges. Current standard of care for neonates of $H B s A g$-positive mothers includes active and passive immunization against $\mathrm{HBV}$ within $12 \mathrm{~h}$ after delivery. Unfortunately, in many cases the generated protective immunity is not sufficient, evidenced by $5-10 \%$ of breakthrough HBV infections [14]. Furthermore, certain patient groups, such as neonates, elderly people or immunosuppressed patients, respond only weakly to the available HBV vaccines $[1,15]$. In countries with limited healthcare resources, a single vaccination right after birth would be a promising approach to overcome these issues. Thus, the development of an optimized vaccine formulation is still of great importance.

\section{Advantages of nanoparticulate vaccine constructs}

The outlined limitations of HBV vaccines drive the growing interest in the production of tailor-made immunization strategies. Virus-derived nanoparticles are mainly attractive for innovative vaccine formulations, enabling the development of robust and biodegradable protein nanocapsules made of the pathogen-specific antigen. Therefore, polymeric $H B s A g$-nanocapsules ( $H B s A g$-NCs) were synthesized using the so-called miniemulsion process exclusively out of the recombinant $H B s A g$-VLPs [14]. The preparation of nanocontainers with a hydrophilic core from water-in-oil miniemulsions and their subsequent transfer to aqueous medium is of special importance, since it enables the efficient encapsulation of hydrophilic payloads in large quantities. For the development of vaccine formulations against hepatitis $\mathrm{C}$ virus $(\mathrm{HCV})$ infection the NS5A protein was used [16]. The major advantages of synthetic particulate vaccines are the size and surface properties of the nanocapsules that can be adjusted accurately [17], the co-administration of vaccine-antigen and -adjuvant and thus the possibility to target certain cells of the immune system [18,19].

“...this particulate antigen-adjuvant platform has the potential to be used as a single neonatal vaccination with a more robust immune response compared with current vaccination strategies."

Uptake of antigen by APCs, especially dendritic cells (DCs) and intrahepatic macrophages, such as Kupffer cells, depends on several antigen-associated properties. In previous studies we could show that the functionalization of NCs with the FDA-approved adjuvant monophosphoryl lipid A (MPLA), a Toll-like receptor 4 agonist, accelerates the uptake by nonparenchymal liver cells, preferably by DCs and Kupffer cells $[14,20]$. In addition, coating of MPLA onto the nanocapsule surface leads to a significant reduction of the required adjuvant [21]. Furthermore, targeting of DCs with $H B s A g$ and MPLA adjuvant resulted in an enhanced uptake of the antigen and simultaneous maturation of these cells and secretion of proinflammatory cytokines, especially IL-12 [14]. Thus, MPLA promotes a $\mathrm{T}_{\mathrm{H}} 1$-directed immune response with the potential to prime antigen-specific $T$ cells and induce robust cellular immunity.

\section{Specific advantages of HBV nanocapsules}

The organ-specific distribution is affected by the route of vaccine administration and by a suitable chemical formulation of polymeric nanocapsules. Intravenous administration of particulate formulations results in a liver-specific uptake by targeting liver immunogenic cells. Thus, the primary entry side of viral infections, such as HBV and HCV, can be targeted subsequently resulting in an intrahepatic immune response. In recent studies, the intravenous immunization of mice with NS5A-NCs induced intrahepatic antigen-specific $\mathrm{CD}^{+}$ T-cell immune responses determined by the secretion on IFN- $\gamma$ and IL-2 [16]. The induction of $\mathrm{T}_{\mathrm{H}} 1$-responses, an essential prerequisite for the treatment of chronic infections, was additionally achieved through the inclusion of MPLA and other adjuvants, such as R848, in combination with IFN- $\gamma$. We could show that a significant increase of T-cell proliferation and their antigen-specific release of IFN- $\gamma$ was induced by pulsing neonatal monocyte-derived DCs with $H B s A g-M P L A-N C s$ plus IFN- $\gamma$ [14]. Thus, this particulate antigen-adjuvant platform has the potential to be used as a single neonatal vaccination with a more robust immune response compared with current vaccination strategies. Remarkably, immunization with currently FDA-approved HBV vaccines, consisting of $H B s A g-V L P s$ adsorbed onto alum, results in insufficient protection against $\mathrm{HBV}$ infection within the immunized pediatric population in $10 \%$ of cases [15]. In addition, synthetic nanoparticle constructs might provide the opportunity to increase the overall vaccine stability. The possibility to lyophilize polymeric particle formulations omits the necessity to maintain the cold chain and promises extended durability, thus, representing a more feasible approach for countries with infrastructural challenges.

\section{Conclusion}

In conclusion, polymeric antigen-based nanocapsules are particularly attractive for improving vaccines as an antigen-adjuvant delivery system to target key cells of the immune system, particularly in the liver. Thus, the synthesis of safe and immunogenic nanocapsules as well as the induction of potent and long-lasting $\mathrm{T}_{\mathrm{H}}$ 1-directed immune responses through single dose 
vaccination is a promising approach to overcome the outlined limitations.

\section{Financial \& competing interests disclosure}

The authors have no relevant affiliations or financial involvement with any organization or entity with a financial inter-

\section{References}

1 Orlando R, Foggia M, Maraolo AE et al. Prevention of hepatitis B virus infection: from the past to the future. Eur. J. Clin. Microbiol. Infect. Dis. 34(6), 1059-1070 (2015).

2 Thoelen S, De Clercq N, Tornieporth N. A prophylactic hepatitis B vaccine with a novel adjuvant system. Vaccine 19(17-19), 2400-2403 (2001).

3 Zhao Q, Wang Y, Abraham D, Towne V, Kennedy R, Sitrin RD. Real time monitoring of antigenicity development of HBsAg virus-like particles (VLPs) during heat- and redoxtreatment. Biochem. Biophys. Res. Commun. 408(3), 447-453 (2011).

4 Kee GS, Jin J, Balasundaram B, Bracewell DG, Pujar NS, Titchener-Hooker NJ. Exploiting the intracellular compartmentalization characteristics of the $S$. cerevisiae host cell for enhancing primary purification of lipid-envelope virus-like particles. Biotechnol. Prog. 26(1), 26-33 (2010).

5 Roldao A, Mellado MC, Castilho LR, Carrondo MJ, Alves PM. Virus-like particles in vaccine development. Expert Rev. Vaccines 9(10), 1149-1176 (2010).

6 Saade F, Honda-Okubo Y, Trec S, Petrovsky N. A novel hepatitis B vaccine containing Advax, a polysaccharide adjuvant derived from delta inulin, induces robust humoral and cellular immunity with minimal reactogenicity in preclinical testing. Vaccine 31(15), 1999-2007 (2013).

7 Pfaar O, Cazan D, Klimek L, Larenas-Linnemann D, Calderon MA. Adjuvants for immunotherapy. Curr. Opin. Allergy Clin. Immunol. 12(6), 648-657 (2012).

8 Mbow ML, De Gregorio E, Ulmer JB. Alum's adjuvant action: grease is the word. Nat. Med. 17(4), 415-416 (2011).

9 Marrack P, McKee AS, Munks MW. Towards an understanding of the adjuvant action of aluminium. Nat. Rev. Immunol. 9, 287-293 (2009).

10 Kool M, Fierens K, Lambrecht BN. Alum adjuvant: some of the tricks of the oldest adjuvant. J. Med. Microbiol. 61(Pt 7), 927-934 (2012).

11 Ferrari C. HBV and the immune response. Liver Int. 35(Suppl. 1), 121-128 (2015). est in or financial conflict with the subject matter or materials discussed in the manuscript. This includes employment, consultancies, honoraria, stock ownership or options, expert testimony, grants or patents received or pending, or royalties.

No writing assistance was utilized in the production of this manuscript.

12 Das A, Maini MK. Innate and adaptive immune responses in hepatitis B virus infection. Digestive Diseases 28(1), 126-132 (2010).

13 Lemoine M, Nayagam S, Thursz M. Viral hepatitis in resource-limited countries and access to antiviral therapies: current and future challenges. Future Virol. 8(4), 371-380 (2013).

14 Pietrzak-Nguyen A, Piradashvili K, Fichter M et al. MPLA-coated hepatitis B virus surface antigen ( $\mathrm{HBsAg}$ ) nanocapsules induce vigorous $\mathrm{T}$ cell responses in cord blood derived human T cells. Nanomedicine 12(8), 2383-2394 (2016).

15 Jafarzadeh A, Shokri F. TH1 and TH2 responses are influenced by HLA antigens in healthy neonates vaccinated with recombinant hepatitis B vaccine. Iran J. Allergy Asthma Immunol. 11(4), 308-315 (2012).

16 Fichter M, Piradashvili K, Pietrzak-Nguyen A et al. Polymeric hepatitis $\mathrm{C}$ virus non-structural protein $5 \mathrm{~A}$ nanocapsules induce intrahepatic antigen-specific immune responses. Biomaterials 108, 1-12 (2016).

17 Baier G, Fichter M, Kreyes A et al. Glutathione responsive hyaluronic acid nanocapsules obtained by bioorthogonal interfacial "click" reaction. Biomacromolecules 17(1), 148-153 (2016).

18 Blander JM, Medzhitov R. Toll-dependent selection of microbial antigens for presentation by dendritic cells. Nature 440(7085), 808-812 (2006).

19 Tacken PJ, Zeelenberg IS, Cruz LJ et al. Targeted delivery of TLR ligands to human and mouse dendritic cells strongly enhances adjuvanticity. Blood 118(26), 6836-6844 (2011).

20 Pietrzak-Nguyen A, Fichter M, Dedters M et al. Enhanced in vivo targeting of murine nonparenchymal liver cells with monophosphoryl lipid A functionalized microcapsules. Biomacromolecules 15, 2378-2388 (2014).

21 Fichter M, Dedters M, Pietrzak-Nguyen A et al. Monophosphoryl lipid A coating of hydroxyethyl starch nanocapsules drastically increases uptake and maturation by dendritic cells while minimizing the adjuvant dosage. Vaccine 33, 838-846 (2015). 